\title{
Probing ultrafast photo-induced dynamics of the exchange energy in a Heisenberg antiferromagnet
}

\author{
G. Batignani'1,2, D. Bossini ${ }^{3}$, N. Di Palo', C. Ferrante', E. Pontecorvo', G. Cerullo4, A. Kimel ${ }^{3}$ \\ and T. Scopigno ${ }^{1,5 \star}$
}

\begin{abstract}
Manipulating the macroscopic phases of solids using ultrashort light pulses has resulted in spectacular phenomena, including metal-insulator transitions ${ }^{1-3}$, superconductivity ${ }^{4}$ and subpicosecond modification of magnetic order ${ }^{5}$. The development of this research area strongly depends on the understanding and optical control of fundamental interactions in condensed matter, in particular the exchange interaction. However, disentangling the timescales relevant for the contributions of the exchange interaction and spin dynamics to the exchange energy, $E_{\text {ex }}$ is a challenge. Here, we introduce femtosecond stimulated Raman scattering to unravel the ultrafast photoinduced dynamics of magnetic excitations at the edge of the Brillouin zone. We find that femtosecond laser excitation of the antiferromagnet $\mathrm{KNiF}_{3}$ triggers a spectral shift of the two-magnon line, the energy of which is proportional to $E_{\mathrm{ex}}$. By unravelling the photo-induced modification of the twomagnon line frequency from a dominating nonlinear optical effect, we find that $E_{\text {ex }}$ is increased by the electromagnetic stimulus.

Magnetic order is a macroscopic manifestation of the quantum phenomenon of exchange coupling between spins. Under thermodynamic equilibrium conditions, the energy of the interaction is conventionally described in the Heisenberg-Dirac form
\end{abstract}

$$
\langle\hat{H}\rangle=E_{\mathrm{ex}}=\frac{1}{2} \sum_{a \neq b} J_{a b}\left\langle\hat{\mathbf{S}}_{a} \cdot \hat{\mathbf{S}}_{b}\right\rangle
$$

where $E_{\mathrm{ex}}$ is the exchange energy, $J_{a b}$ is the exchange interaction, and the last term is the correlation function between spins on neighbouring sites $a$ and $b$.

Despite the enormous number of experiments reporting on the optical control of spins ${ }^{5}$, there are very few studies of the ultrafast photo-induced dynamics of the exchange interaction ${ }^{6,7}$. One of the reasons for the popularity of spin dynamics studies is the possibility to use all-optical techniques, which can be applied easily to a broad class of materials. However, the studies that have succeeded in revealing the dynamics of the exchange energy $E_{\text {ex }}$ make use of the rather demanding and less flexible technique of time- and spinresolved photoelectron spectroscopy. Despite its complexity, photoelectron spectroscopy has proven to be very powerful when applied to metal surfaces ${ }^{6,7}$. In these materials, however, ultrafast laser excitation also triggers electronic dynamics, which defines the dynamics of $J$, and demagnetization ${ }^{8,9}$, which is related to the spin correlation function, both on the femtosecond timescale. So, disentangling the dynamics of the two contributions appearing in equation (1) is challenging. In some semiconductors, the overall dynamics of the $d-f$ exchange energy has been monitored by measuring the transient optical properties ${ }^{10}$. On the other hand, the scenario in dielectrics is markedly different. Although the timescale of the charges' response is still basically limited by the duration of a femtosecond stimulus, demagnetization occurs over a timescale as long as $100 \mathrm{ps}$ after photoexcitation ${ }^{11}$. Assessing the capability of light to probe the exchange energy at the femtosecond timescale in such cases would therefore allow the dynamics of $J_{a b}$ to be distinguished. This would greatly facilitate a systematic investigation of dynamic exchange in a broad class of materials, thus providing an excellent future for fundamental studies of ultrafast magnetism beyond the adiabatic approximation and the Heisenberg model. Critically, an experimental scheme able to probe the ultrafast light-induced modification of $J_{a b}$ has not yet been developed, and is the aim of the present work.

The contribution of the exchange interaction to magnetic dispersion $^{12}$ is dominant at the zone edges. A convenient way to access this region is provided by 'two-magnon (2M) light scattering', conventionally described in terms of couples of interacting magnons whose wavevectors lie near the edges of the first Brillouin zone, as determined by the law of conservation of momentum and by the magnon density of states, which typically peaks far from the zone centre ${ }^{12-15}$.

Spontaneous Raman (SR) spectroscopy ${ }^{16-19}$ has been widely used to measure the frequency of the $2 \mathrm{M}$ line $\left(\omega_{2 \mathrm{M}}\right)$ which represents the increase of $E_{\mathrm{ex}}$ relative to the ground-state exchange energy $E_{\mathrm{ex}}^{0}$, corresponding to a completely ordered spin lattice. Such an increase, in a Heisenberg antiferromagnet, is generated by two spin flip events occurring on neighbouring sites, one for each magnetic sublattice ${ }^{12}$. Accordingly, from equation (1) it follows that

$$
\hbar \omega_{2 \mathrm{M}}=E_{\mathrm{ex}}-E_{\mathrm{ex}}^{0}=\frac{2 n S-1}{S^{2}} J_{a b}\left\langle\hat{\mathbf{s}}_{a} \cdot \hat{\mathbf{s}}_{b}\right\rangle=\frac{2 n S-1}{N} E_{\mathrm{ex}}
$$

where $S$ is the spin of the system, $n$ is the number of nearest neighbours, and $N$ is the total number of sites. The process underlying the $2 \mathrm{M}$ mode, usually denoted as exchange scattering, is Raman-allowed by a light-induced modification of the exchange interaction ${ }^{12,14,20}$.

Although SR spectroscopy is an ideal tool to map an equilibrium structure, it can also be used as a probe in time-resolved experiments to address transient phases ${ }^{21}$. It has, however, a critical shortcoming when addressing subpicosecond phenomena. Specifically, it is subject to a time-energy resolution restriction dictated by the time-bandwidth product of the light pulse, which is limited by $\Delta E \Delta t \geq 15 \mathrm{~cm}^{-1}$ ps (ref. 22).

In this Letter, we circumvent this limitation by taking advantage of the unrestricted time precision and energy resolution of femtosecond stimulated Raman spectroscopy (FSRS) ${ }^{23}$, which we use to

'Dipartimento di Fisica, Universitá di Roma 'La Sapienza', Roma I-00185, Italy. ${ }^{2}$ Dipartimento di Scienze Fisiche e Chimiche, Universitá degli Studi dell'Aquila, L'Aquila I-67100, Italy. ${ }^{3}$ Radboud University Nijmegen, Institute for Molecules and Materials, Nijmegen, AJ 6525, The Netherlands. ${ }^{4}$ IFN-CNR, Dipartimento di Fisica, Politecnico di Milano, Piazza L. da Vinci 32, Milano 20133, Italy. ${ }^{5}$ Center for Life Nano Science @Sapienza, Istituto Italiano di Tecnologia, 295 Viale Regina Elena, Roma I-00161, Italy. *e-mail: tullio.scopigno@phys.uniroma1.it 
a
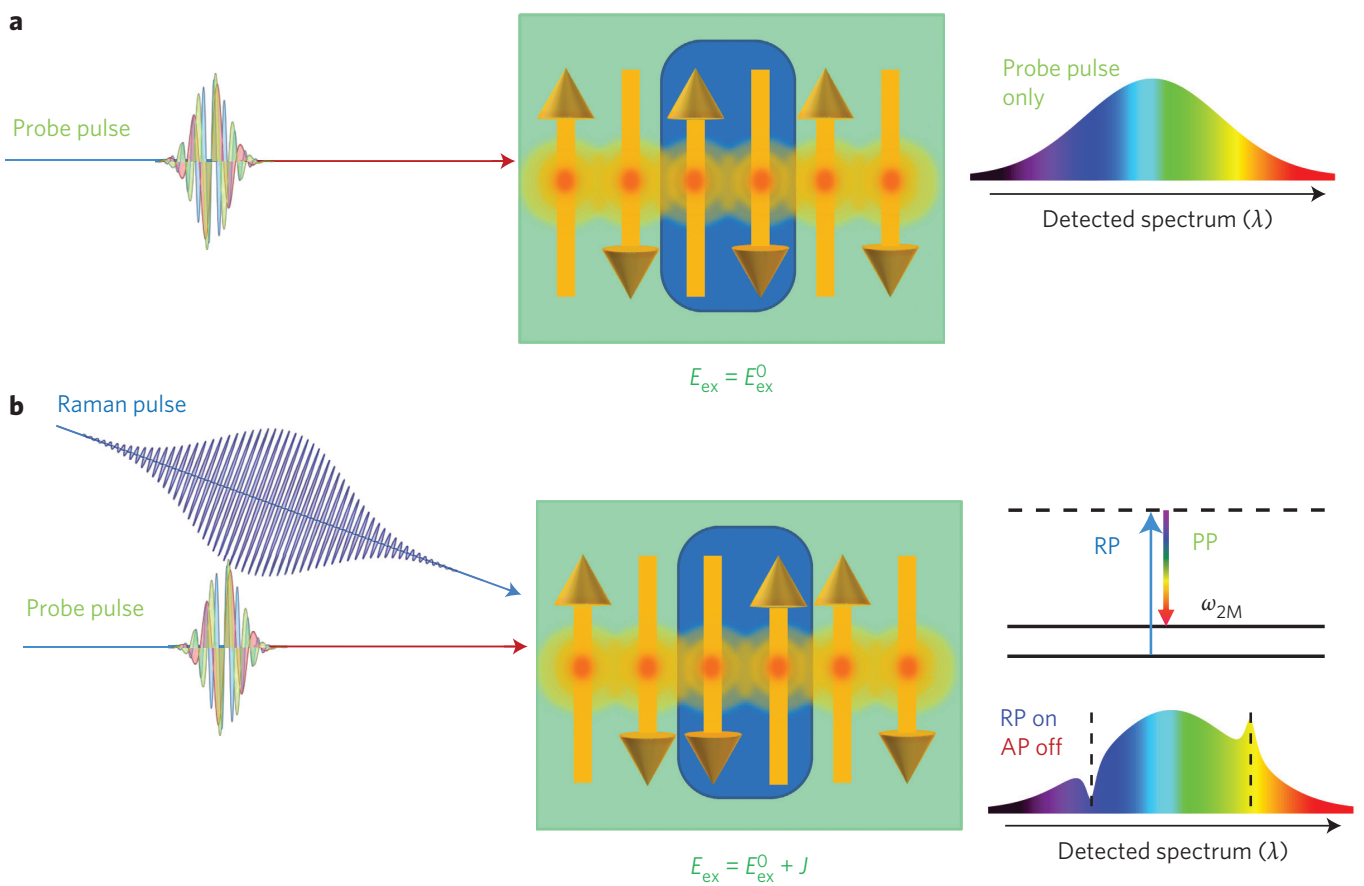

c
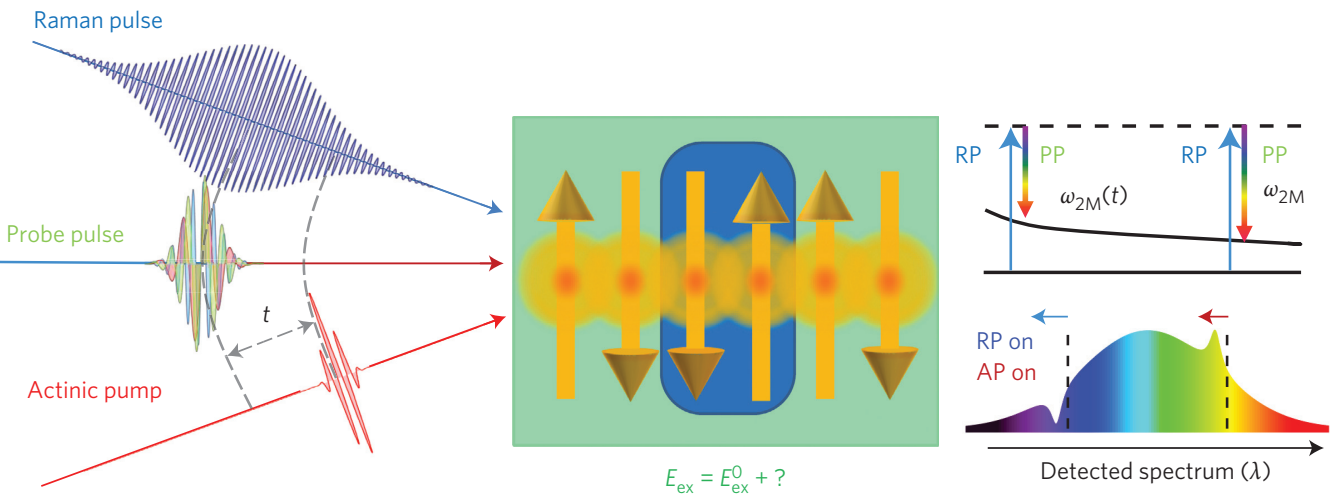

Figure 1 | Concept of the FSRS experiment on $\mathrm{KNiF}_{3}$. $\mathbf{a}, \mathrm{A}$ femtosecond white-light continuum (WLC) acts as a probe pulse (PP). $\mathbf{b}$, Superposition of a picosecond narrow-bandwidth Raman pulse (RP) with the PP generates Raman-induced coherences, read out on top of the WLC as (positive) gain and (negative) losses. c, An actinic pump excitation (AP) modifies the exchange energy.

track the dynamics of the $2 \mathrm{M}$ line of the cubic perovskite $\mathrm{KNiF}_{3}$ upon ultrashort optical excitation. The basics of our FSRS set-up have been described elsewhere ${ }^{24,25}$, and the details relevant to this experiment are provided in the Methods. A sketch of the concept is presented in Fig. 1, where the probe field is a femtosecond white-light pulse (Fig. 1a), which, in the presence of a spatially and temporally overlapped narrowband picosecond field (the Raman pulse) stimulates the $2 \mathrm{M}$ excitation by triggering the spinflip events. This results in a net increase of $E_{\mathrm{ex}}$, which, for a simplified one-dimensional case (a linear chain with $S= \pm 1 / 2$ ), is $\Delta E_{\mathrm{ex}}=J$, as can be easily derived from equation (2). The resulting stimulated Raman scattering (SRS) signal arises as a modification of the probe pulse transmissivity, induced by the third-order Raman susceptibility, which generates a polarization $P^{(3)}(\omega)$ on top of the probe field $E_{\mathrm{P}}(\omega)$ (Fig. 1b). The corresponding stimulated Raman gain is given by $\operatorname{SRG}(\omega) \propto \mathfrak{J}\left(P^{(3)}(\omega) / E_{\mathrm{P}}(\omega)\right)$. The addition of an ultrashort actinic pump pulse to this probing scheme allows the photo-induced modification of the stimulated Raman spectrum to be investigated, and hence $E_{\text {ex }}$ (Fig. 1c).

Figure 2 shows the excellent agreement of the 2 magnon line (2ML) frequency and linewidth as measured by SR and SRS spectroscopies at different temperatures, which is well described by equation (2) with $S=1$ and $n=6$ (refs 20,27). The blueshifted (relative to the Raman pulse frequency) component is a Raman loss (RL), antisymmetric to a redshifted Raman gain (RG), which has no spontaneous (anti-Stokes) counterpart ${ }^{26}$.

The light-induced modification of the $2 \mathrm{M}$ peak frequency, in the presence of an ultrashort off-resonance actinic pump pulse, is shown in Fig. 3 for different delays with respect to the probe pulse. The position of the Raman line was estimated by fitting the data with a pseudo-Voigt function (Supplementary Section 'Fit procedure and data treatment' and Supplementary Fig. 1). Remarkably, the spectral dynamics of the RG and RL are not symmetric, as sketched in the inset. For positive time delays the RG wavelength undergoes a blueshift corresponding to a lightinduced decrease in the $2 \mathrm{M}$ frequency, and then fully recovers its unperturbed position in less than $150 \mathrm{fs}$.

Critically, we also observe a blueshift for the RL peak corresponding to an increase in the $2 \mathrm{M}$ frequency, which is at odds with a purely dynamical effect, that is, a light-induced modification of the $2 \mathrm{M}$ frequency. Moreover, such a peak shift is also observed at negative time delays, where the $2 \mathrm{M}$ frequency is still unaffected by the actinic pulse. This behaviour suggests the dominance of a purely optical, off-resonant fifth-order nonlinear process (that is, 

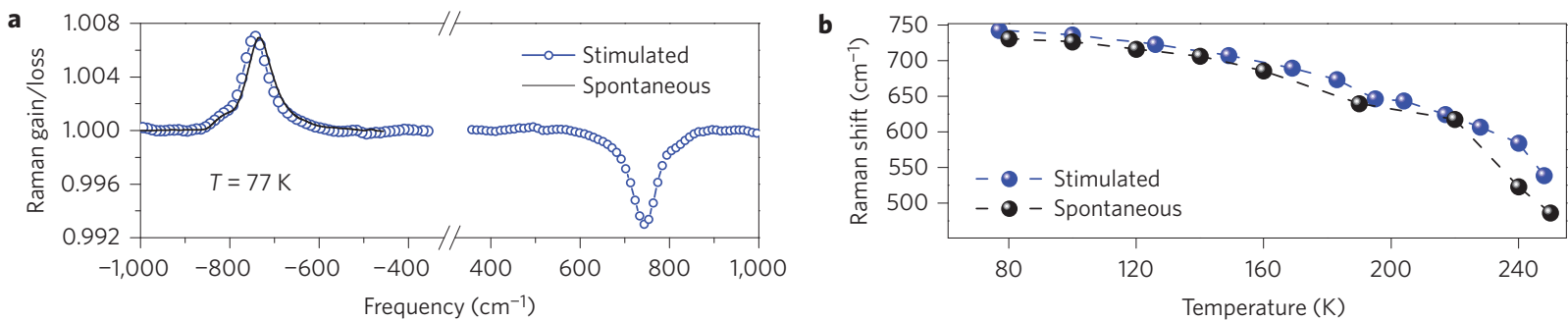

Figure 2 | SRS measurements on $\mathrm{KNiF}_{3}$. $\mathbf{a}$, Background-subtracted Raman loss (RL) and Raman gain (RG) stimulated Raman spectra, obtained using a narrowband Raman pulse and a broadband probe pulse, compared to a (scaled) Stokes side spontaneous Raman measurement at $T=77 \mathrm{~K}$. The redshifted (relative to the Raman pulse wavelength) component is a RG, which is in excellent agreement with the previously measured spontaneous (Stokes) Raman spectrum of the $2 \mathrm{M}$ mode ${ }^{16-19}$. The blueshifted component is a RL, antisymmetric to the RG with respect to the Raman pulse frequency, which has no spontaneous (anti-Stokes) counterpart ${ }^{26}$. $\mathbf{b}$, Softening of the $2 \mathrm{M}$ line with increasing temperature, as measured by SRS and SR spectroscopies.

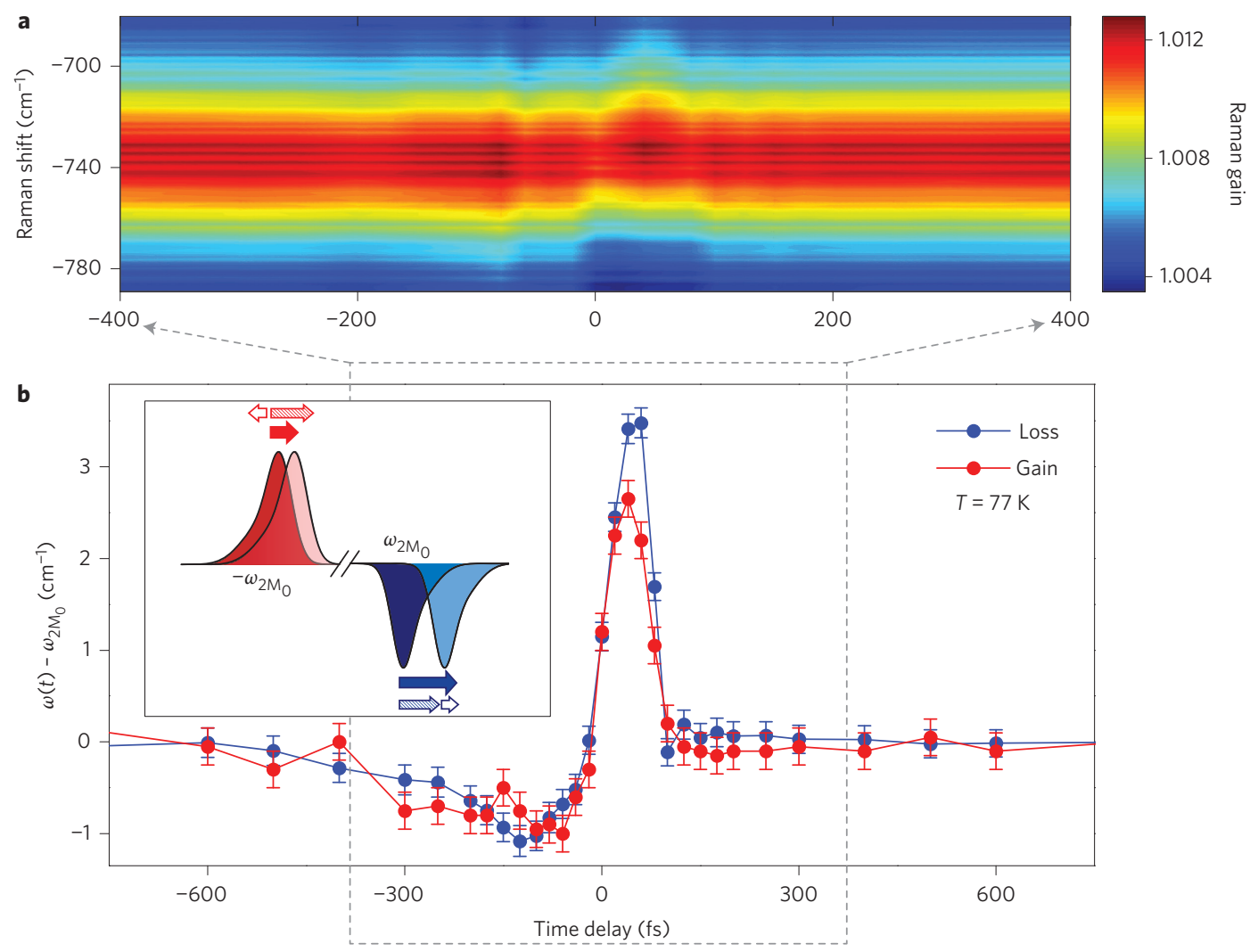

Figure 3 | Experimental peak shift of the $2 \mathrm{M}$ line from the RG and RL components of the stimulated Raman spectra. $\mathbf{a}$, 2M line FSRS spectra on the red side of the Raman pulse at different time delays. The colourmap shows a variation of the peak position during the time overlap condition among the three different pulses. The measurements were performed with horizontal actinic pump polarization, orthogonal to the other two beams, with $1.9 \mathrm{eV}$ photon energy and $60 \mathrm{~mJ} \mathrm{~cm}^{-2}$ fluence. $\mathbf{b}$, Photo-induced evolution of the Raman shift of the $2 \mathrm{M}$ line as a function of actinic pump delay in the FSRS spectra, for both gain and loss sides. Error bars indicate the $90 \%$ confidence interval of the best fitted peak position. Inset: the line shape of the $2 \mathrm{M} \mathrm{Raman}$ shift for RG (red) and RL (blue) features is ascribed to the sum (thick arrows) of two effects with opposite symmetries: exchange energy modification (thin filled arrows) and a six-wave mixing process (thin open arrows). $\omega_{2 \mathrm{M}_{0}}$ indicates the position of the $2 \mathrm{M}$ mode peak in the absence of actinic photoexcitation (equation (3) in the Methods). The peak shifts, detected under time overlap conditions, are different for the RG and RL. The photo-induced modification of the exchange energy can be isolated by taking the difference between the frequency shifts of the RG and RL peaks.

six-wave mixing), occurring when the Raman pulse overlaps in time with the actinic pump ${ }^{28}$.

This process is a perturbation, by the actinic pulse, of the Raman free induction decay and extends over the dephasing time of the $2 \mathrm{M}$ mode. To validate our hypothesis we performed a control FSRS experiment under similar conditions on a non-magnetic system, namely liquid cyclohexane, which can be used as a reference benchmark subject to the non-resonant six-wave mixing process only. As expected, both RG and RL undergo a blueshift, as in $\mathrm{KNiF}_{3}$ (Supplementary Fig. 2). However, in the cyclohexane case, RG and RL shift by the same absolute value, but this is not the case in $\mathrm{KNiF}_{3}$.

Specifically, as reported in Fig. 3, for $\mathrm{KNiF}_{3}$ the RG and RL shifts for a given positive time delay are different, suggesting the possible presence of a net, genuine photo-induced transient increase in $\omega_{2 \mathrm{M}}$ contributing to the response as a symmetric shift (blueshift of the RL and redshift of the RG), quantified by $\Delta \omega_{2 \text { Mmeas }}(t)=(1 / 2)\left[\left|\Delta \omega_{\mathrm{RL}}\right|-\left|\Delta \omega_{\mathrm{RG}}\right|\right]$.

The derivative-like line shape of the $2 \mathrm{M}$ Raman shift consists therefore of two concurring effects: a light-induced modification 
of the exchange energy and a six-wave mixing process. The former is only present at positive time delays and is represented by the difference between the blue and red lines, which are reported in Fig. 3b. As elucidated in the inset of Fig. 3b, the different magnitudes of the absolute frequency shift measured at positive time delays can be explained by the opposite symmetry of the wave-mixing process with respect to the net photo-induced effect on $E_{\text {ex }}(t)$.

Remarkably, the entire dynamics manifests itself in a temporal window narrower than $100 \mathrm{fs}$, demonstrating the potential brought about by the FSRS time-energy resolution for condensed matter studies. An important point to note, however, is the precise relation between the measured frequency shift $\Delta \omega_{2 \text { Mmeas }}(t)$ and the true dynamics of the $2 \mathrm{M}$ mode $\Delta \omega_{2 \mathrm{M}}(t)$ at such ultrafast regimes. This heavily depends on the ratio between the timescale of the transient variation of $E_{\mathrm{ex}}, \tau$ and the dephasing time of the induced coherence $\Gamma^{-1}$, corresponding to the inverse of the Raman linewidth.

Specifically, under the condition $\tau \gg \Gamma^{-1}$, the FSRS spectrum consists of narrow gain (and loss) features tracking the instantaneous dynamics; that is, $\Delta \omega_{2 \mathrm{Mmeas}}(t)$ and $\Delta \omega_{2 \mathrm{M}}(t)$ would be identical. When the dynamics (wavepacket motion) occurs on a timescale $\tau$ comparable with the dephasing time $\left(\tau \approx \Gamma^{-1}\right)$, the link between the instantaneous frequency and the lineshape is not straightforward, to the extent that the spectral response can even become dispersive ${ }^{29,30}$. The case of interest here is even more critical, being $\tau \ll \Gamma^{-1} \quad(\tau \approx 40 \mathrm{fs}$, evaluated as the full-width at halfmaximum (FWHM) of $\Delta \omega_{2 \text { Mmeas }}(t)$ in Fig. 3 and $\Gamma^{-1} \approx 500 \mathrm{fs}$ from Fig. 2a).

Under these circumstances, the maximum value of the photoinduced modification of $\omega_{2 \mathrm{M}}(t)$ corresponds to an increase by $\sim 3 \%$ of the unperturbed Raman shift (see Methods) and therefore of the exchange energy (equation (2)). This is in contrast with the results reported in metals ${ }^{6,7}$, where the ultrafast quenching of the energy splitting between the spin majority and spin minority bands reveals a light-induced reduction of the exchange energy. However, it is not surprising that the effect of an optical stimulus on a dielectric medium is different, considering that in this case the huge dissipations due to the absorption of light by the free electrons are absent. Moreover, we note that a recent theoretical study predicts a photo-induced increase in the exchange interaction of the same order of our estimation, for a super-exchange dielectric antiferromagnet under the condition of off-resonant excitation ${ }^{31}$. We therefore ascribe the observed modification of the exchange energy to the microscopic magnetic interactions out of equilibrium, which have been proven to be able to modify the exchange interaction via a light-induced energy shift of the charge transfer transition $^{31,32}$.

In summary, we have demonstrated that ultrafast coherent Raman spectroscopy can provide experimental evidence for sub$100 \mathrm{fs}$ photo-induced dynamics of magnons in the high- $k$ region of the Brillouin zone, direct expression of a light-induced increase in the exchange energy. Our results provide an estimate of the dissipative dynamics of the $2 \mathrm{M}$ line, setting an experimental benchmark for theoretical descriptions of ultrafast laser-induced magnetic dynamics beyond the Heisenberg model. Moreover, we introduce an approach to obtain the real dynamics of a Raman mode from FSRS measurements, on timescales as short as the duration of the pump and probe pulses. We anticipate that an investigation of the dynamics of the $2 \mathrm{M}$ mode triggered by resonant excitation of the charge-transfer transition would reveal different pathways for optical control of the exchange energy.

\section{Methods}

Methods and any associated references are available in the online version of the paper.
Received 12 May 2015; accepted 11 June 2015; published online 13 July 2015

\section{References}

1. Rini, M. et al. Control of the electronic phase of a manganite by mode-selective vibrational excitation. Nature 449, 72-74 (2007).

2. Ichikawa, H. et al. Transient photoinduced 'hidden' phase in a manganite. Nature Mater. 10, 101-105 (2011).

3. de Jong, S. et al. Speed limit of the insulator-metal transition in magnetite. Nature Mater. 12, 882-886 (2013).

4. Fausti, D. et al. Light-induced superconductivity in a stripe-ordered cuprate. Science 331, 189-191 (2011).

5. Kirilyuk, A., Kimel, A. V. \& Rasing, T. Ultrafast optical manipulation of magnetic order. Rev. Mod. Phys. 82, 2731-2784 (2010).

6. Rhie, H.-S., Dürr, H. A. \& Eberhardt, W. Femtosecond electron and spin dynamics in Ni/W(110) films. Phys. Rev. Lett. 90, 247201 (2003).

7. Carley, R. et al. Femtosecond laser excitation drives ferromagnetic gadolinium out of magnetic equilibrium. Phys. Rev. Lett. 109, 057401 (2012).

8. Koopmans, B. et al. Explaining the paradoxical diversity of ultrafast laserinduced demagnetization. Nature Mater. 9, 259-265 (2010).

9. Beaurepaire, E., Merle, J.-C., Daunois, A. \& Bigot, J.-Y. Ultrafast spin dynamics in ferromagnetic nickel. Phys. Rev. Lett. 76, 4250-4253 (1996).

10. Subkhangulov, R. R. et al. All-optical manipulation and probing of the $d-f$ exchange interaction in EuTe. Sci. Rep. 4, 4368-4372 (2014).

11. Bossini, D., Kalashnikova, A. M., Pisarev, R. V., Rasing, T. \& Kimel, A. V. Controlling coherent and incoherent spin dynamics by steering the photoinduced energy flow. Phys. Rev. B 89, 060405(R) (2014).

12. Cottam, M. G. \& Lockwood, D. J. Light Scattering in Magnetic Solids (Wiley, 1986).

13. Elliott, R. J. \& Thorpe, M. F. The effects of magnon-magnon interaction on the two-magnon spectra of antiferromagnets. J. Phys. C 2, 1630-1643 (1969).

14. Cottam, M. G. Theory of two-magnon Raman scattering in antiferromagnets at finite temperatures. J. Phys. C 5, 1461-1474 (1972).

15. Hayes, W. \& Loudon, R. Scattering of Light by Crystals (Wiley, 1978).

16. Martin, T., Merlin, R., Huffman, D. \& Cardona, M. Resonant two magnon Raman scattering in $\alpha-\mathrm{Fe}_{2} \mathrm{O}_{3}$. Solid State Commun. 22, 565-567 (1977).

17. Lockwood, D. J., Cottam, M. G. \& Baskey, J. H. One- and two-magnon excitations in NiO. J. Magn. Magn. Mater. 104, 1053-1054 (1992).

18. Abdalian, A. T. et al. Magnon Raman scattering in the two-dimensional antiferromagnet $\mathrm{KFeF}_{4}$. J. Magn. Magn. Mater. 104, 1047-1048 (1992).

19. Fleury, P. A., Porto, S. P. S., Cheesman, L. E. \& Guggenheim, H. J. Light scattering by spin waves in $\mathrm{FeF}_{2}$. Phys. Rev. Lett. 17, 84-87 (1966).

20. Chinn, S. R., Zeiger, H. J. \& O'Connor, J. R. Two-magnon Raman scattering and exchange interactions in antiferromagnetic $\mathrm{KNiF}_{3}$ and $\mathrm{K}_{2} \mathrm{NiF}_{4}$ and ferrimagnetic $\mathrm{RbNiF}_{3}$. Phys. Rev. B 3, 1709-1735 (1971).

21. Fausti, D., Misochko, O. \& van Loosdrecht, P. Ultrafast photoinduced structure phase transition in antimony single crystals. Phys. Rev. B 80, 161207(R) (2009).

22. Rullière, C. (ed.) Femtosecond Laser Pulses: Principles and Experiments (Springer, 2005)

23. McCamant, D. W., Kukura, P., Yoon, S. \& Mathies, R. A. Femtosecond broadband stimulated Raman spectroscopy: apparatus and methods. Rev. Sci. Instrum. 75, 4971-4980 (2004).

24. Pontecorvo, E. et al. Femtosecond stimulated Raman spectrometer in the 320-520 nm range. Opt. Express 19, 1107-1112 (2011).

25. Pontecorvo, E., Ferrante, C., Elles, C. G. \& Scopigno, T. Spectrally tailored narrowband pulses for femtosecond stimulated Raman spectroscopy in the range 330-750 nm. Opt. Express 21, 6866-6872 (2013).

26. Harbola, U., Umapathy, S. \& Mukamel, S. Loss and gain signals in broadband stimulated-Raman spectra: theoretical analysis. Phys. Rev. A 88, 011801 (2013)

27. Schilbe, P., Ramsteiner, M. \& Rieder, K. H. Two-magnon Raman scattering in $\mathrm{KNiF}_{3}$. A comparison of experiment with Monte Carlo calculations in the ordered and disordered regions. J. Phys. Condens. Matter 9, 4979-4986 (1997).

28. Batignani, G., Fumero, G., Mukamel, S. \& Scopigno, T. Energy flow between spectral components in 2D broadband stimulated Raman spectroscopy. Phys. Chem. Chem. Phys. 17, 10454-10461 (2015).

29. Mukamel, S. \& Biggs, J. D. Communication: comment on the effective temporal and spectral resolution of impulsive stimulated Raman signals. J. Chem. Phys. 134, 161101 (2011).

30. Fingerhut, B. P., Dorfman, K. E. \& Mukamel, S. Probing the conical intersection dynamics of the RNA base uracil by UV-pump stimulatedRaman-probe signals; $a b$ initio simulations. J. Chem. Theory Comput. 10, 1172-1188 (2014)

31. Mikhaylovskiy, R. V. et al. Inverse magneto-refraction as a mechanism for laser modification of spin-spin exchange parameters and subsequent terahertz emission from iron oxides. Preprint at http://arxiv.org/abs/1412.7094 (2014)

32. Secchi, A., Brener, S., Lichtenstein, A. \& Katsnelson, M. Non-equilibrium magnetic interactions in strongly correlated systems. Ann. Phys. 333, 221-271 (2013). 


\section{Acknowledgements}

The authors thank R.V. Pisarev for providing the sample, P.H.M. van Loosdrecht and A. Caretta for performing spontaneous Raman measurements and Th. Rasing for continuous support. S. Mukamel is acknowledged for critical reading of the manuscript and useful insights. This research was partially supported by the Nederlandse Organisatie voor Wetenschappelijk Onderzoek (NWO), de Stichting voor Fundamenteel Onderzoek der Materie (FOM), the European Research Council (ERC) under the European Union's Seventh Framework Program (FP7/2007-2013) grant agreements no. 207916 (Femtoscopy) and no. 257280 (Femtomagnetism).

\section{Author contributions}

D.B., G.C., A.K. and T.S. conceived the project. D.B. and A.K. suggested the idea to probe the exchange energy via the $2 \mathrm{M}$ line. G.B., N.D.P., C.F., E.P. and T.S. designed the experiment and the method to interpret the FSRS data to obtain the 2ML dynamics. E.P., with assistance from G.B., D.B. and N.D.P., led the experimental activity. G.B., with help from N.D.P. and C.F., performed data analysis and numerical modelling. T.S. directed the research, and wrote the manuscript with G.B. and D.B. All authors discussed the results and implications and commented on the manuscript.

\section{Additional information}

Supplementary information is available in the online version of the paper. Reprints and permissions information is available online at www.nature.com/reprints. Correspondence and requests for materials should be addressed to T.S.

\section{Competing financial interests}

The authors declare no competing financial interests. 


\section{Methods}

Sample. $\mathrm{KNiF}_{3}$ is a cubic perovskite with space group of cubic symmetry $m 3 m$ (ref. 33), with two equivalent $\mathrm{Ni}^{2+}$ sublattices antiferromagnetically coupled below the Neel temperature $T_{\mathrm{N}}=246 \mathrm{~K}$ (ref. 34 ). The sample was a $340-\mu \mathrm{m}$-thick (100) plane-parallel plate.

Experimental set-up. A Ti:sapphire laser was used to generate $3.6 \mathrm{~mJ}, 35 \mathrm{fs}$ pulses at $800 \mathrm{~nm}$, with a $1 \mathrm{kHz}$ repetition rate. A portion of the laser fundamental, driving a two-stage optical parametric amplifier, produced a horizontally polarized $60 \mathrm{fs}$ actinic pump $\left(E_{\mathrm{A}}(t)\right)$ with $1.9 \mathrm{eV}$ photon energy and $60 \mathrm{~mJ} \mathrm{~cm} \mathrm{~cm}^{-2}$ fluence, which was used for photoexcitation. Snapshots of the subsequent $2 \mathrm{M}$ line evolution were taken by broadband SRS, where the simultaneous presence of a narrowband picosecond pulse (Raman pulse, $E_{\mathrm{R}}(t)$ ) and a femtosecond WLC (Raman probe, $E_{\mathrm{P}}(t)$ ) generates vibrational coherence. Raman pulses were synthesized from a second two-stage optical parametric amplifier producing tunable infrared-visible pulses, followed by a spectral compression stage based on frequency doubling in a $25 \mathrm{~mm}$ beta barium borate crystal. Vertically polarized pulses with $2.5 \mathrm{eV}$ photon energy and $10 \mathrm{~cm}^{-1}$ bandwidths were obtained, with $5 \mathrm{~mJ} \mathrm{~cm}{ }^{-2}$ fluence. The femtosecond probe comprised a vertically polarized WLC, generated by focusing the laser fundamental into a sapphire crystal. The Raman features arose as gain (positive or negative) on top of the transmitted WLC, which was frequency dispersed by a spectrometer onto a charge-coupled device. A synchronized chopper was used to block alternating Raman pulse pulses in order to obtain the Raman gain using successive probe pulses, and a second chopper blocked the actinic pump at $250 \mathrm{~Hz}$ to obtain Raman gain spectra with and without actinic excitation. All the pulses were linearly polarized, the WLC and Raman fields were parallel, and the actinic pump field was rotated by $90^{\circ}$ to minimize coherent artefacts.

Calculated FSRS spectra. The FSRS spectral responses reported in Supplementary Fig. 3 were evaluated following the procedure in ref. 29. The signal is given by

$$
S(\omega)=\mathfrak{\Im}\left[P^{(5)}(\omega) E_{P}^{*}(\omega)\right]
$$

where

$$
P^{(5)}(\omega)=\int_{-\infty}^{+\infty} \mathrm{d} t P^{(5)}(t) e^{i \omega t}
$$

Under the present experimental conditions, the dephasing time of the $2 \mathrm{M}$ line $(\sim 500 \mathrm{fs})$ is much shorter than the duration $\tau_{\mathrm{R}}$ of the narrowband Raman pulse ( $\sim 3$ ps), so we assumed a monochromatic Raman pulse $\left(E_{\mathrm{R}}(t)=E_{\mathrm{R}} e^{-i \omega_{\mathrm{R}} t}\right)$, without any loss of generality. Both the actinic pump and the Raman probe pulses are modelled as impulsive, $E_{\mathrm{A}}(t)=E_{\mathrm{A}} \delta(t)$ and $E_{\mathrm{P}}(t)=E_{\mathrm{P}} \delta(t-T)$, for the sake of simplicity. Then, for a three-level system (with the ground state at frequency $\omega_{a}$, vibrational excited state at $\omega_{c}$ and electronic excited state at $\omega_{b}$ ), the nonlinear polarization in the time domain is given by

$$
P^{(5)}(t)=i\left|E_{\mathrm{R}}\right|^{2} E_{P} \frac{\left|\mu_{a b}\right|^{2}\left|\mu_{c b}\right|^{2}}{\left(\omega_{\mathrm{R}}-\omega_{b a}\right)^{2}} \exp \left[-i \omega_{\mathrm{R}}(t-T)+i \int_{T}^{t} \omega_{c a}(\tau) \mathrm{d} \tau-\gamma_{c a}|t-T|\right]
$$

where $\mu_{\mathrm{ij}}, \gamma_{c a}^{-1}$ and $\omega_{c a}(t)$ indicate the dipole matrix amplitudes, the vibrational lifetime and the instantaneous frequency, respectively. The latter is modelled as an exponential function according to equation (3). The relaxed, long-time frequency of the system $\omega_{2 \mathrm{M}}$ is given by the $2 \mathrm{M}$ mode in the absence of photoexcitation $\left(740 \mathrm{~cm}^{-1}\right)$.

Evaluation of $\omega_{2 M}(t)$. As highlighted in the main text, because the condition $\tau=\Gamma^{-1}$ holds for the present case, the system dynamics is exhausted well before the Raman interaction is completed; that is, the Raman process mostly probes the unperturbed state. To quantify the link between $\Delta \omega_{2 \text { Mmeas }}(t)$ and $\omega_{2 M}(t)$ under such circumstances, we used the assumption of an exponential relaxation from $\Delta \omega_{2 \mathrm{M}}+\omega_{2 \mathrm{M}_{0}}$ to $\omega_{2 \mathrm{M}_{0}}$ for the system dynamics ${ }^{29}$ :

$$
\omega_{2 \mathrm{M}}(t)= \begin{cases}\omega_{2 \mathrm{M}_{0}}+\Delta \omega_{2 \mathrm{M}} e^{-t / \tau} & \text { if } t \geq 0 \\ \omega_{2 \mathrm{M}_{0}} & \text { if } t<0\end{cases}
$$

and we found that the largest $\Delta \omega_{2 \text { Mmeas }}$ observed in the probed temporal window corresponds to $\Delta \omega_{2 \mathrm{M}}=20 \mathrm{~cm}^{-1}$. This has to be taken as a lower bound because the value $\tau \approx 40$ fs that we used for the estimate does not account for the finite duration of the pulses. The expected dependence of $\omega_{2 \mathrm{M}}(t)$ as a function of $\tau$ is shown in Supplementary Fig. 3a.

\section{References}

33. Ivanov, Y., Zhurova, E. A., Zhurov, V. V., Tanaka, K. \& Tsirelson, V. Electron density and electrostatic potential of $\mathrm{KNiF}_{3}$ : multipole, orbital and topological analyses of vacuum-camera-imaging plate and four-circle diffractometer data. Acta Crystallogr. B 55, 923-930 (1999).

34. Nouet, J., Zarembowitch, A., Pisarev, R. V., Ferre, J. \& Lecomte, M. Determination of $T_{N}$ for $\mathrm{KNiF}_{3}$ through elastic, magneto-optical, and heat capacity measurements. Appl. Phys. Lett. 21, 161-162 (1972). 